
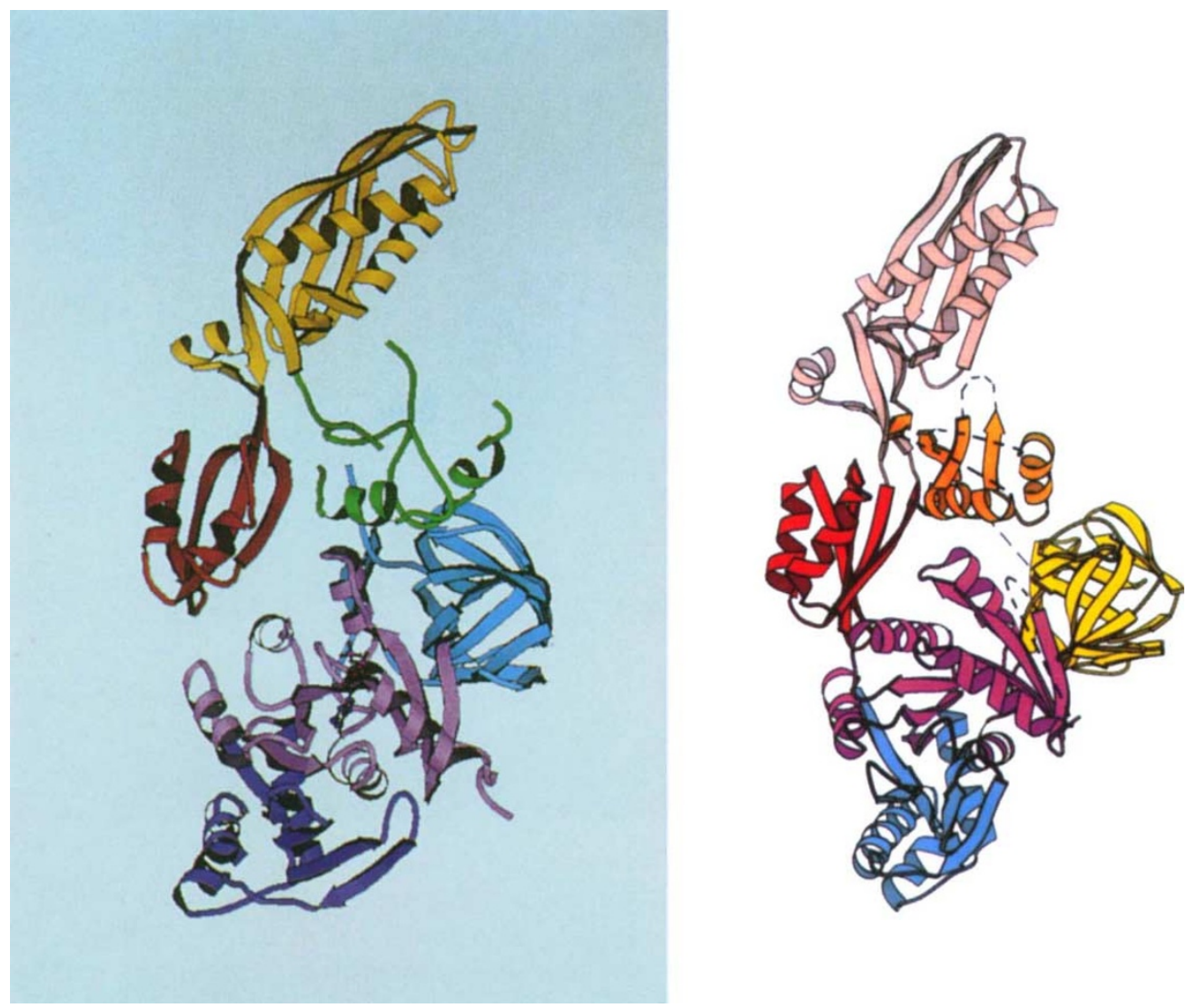

\title{
Helping along peptide production
}

Once the synthesis of a new peptide chain by the ribosome is underway the process can be thought of as a two step cycle. Amino acids, attached to their cognate aminoacyl tRNA, bind to the ribosome and are joined to the end of the growing peptide chain (delivery), then the newly formed end is moved within the ribosome to allow entry of the next amino acid (translocation). Both these reactions are inherent properties of the ribosome; however, they are greatly accelerated by the presence, in bacteria, of two elongation factors: EF-Tu, which mediates delivery, and EF-G, which mediates translocation. Both have eukaryotic counterparts, EF-1 and EF-2.

EF-Tu and EF-G are GTPases, one molecule of GTP being hydrolysed by both for the addition of a single amino acid to the growing peptide chain. They also have overlapping binding sites on the ribosome. It is not surprising then that the recently determined structures of EF-G both with (J.Czworkowski, J.Wang, T.A.Steitz \& P.B.Moore, EMBO J. 13 3661-3668; left) and without bound GDP (A.AEvarsson et al., EMBO J. 13 3669-3677; right) show similarity to that of EF-Tu. In fact EF-G's two amino-terminal domains (purple and blue on the left, violet and yellow on the right) are homologous to two domains of EF-Tu although EF-G contains a 90 residue insertion (dark purple on the left, blue on the right).

In EF-Tu these two domains undergo considerable reorientation between the GTP- and GDPbound forms therefore it is intriguing that EF-G both with and without GDP resembles more closely the GTP-bound form of EF-Tu. This similarity may imply that EF-G does not undergo the same dramatic conformational change as EF-Tu on GTP hydrolysis, or it may reflect the reciprocal nature of the two elongation factors' activity. This conundrum will only be solved by the determination of the GTP-bound form of EF-G. 